\title{
Analysis of signal error rate of time-varying channel effects on ASK band transmission of deep-sea abandoned profiler measuring instrument
}

\author{
Qingwen Li, Yu Zheng, Lei Tian, Guomin Song, Yingsheng Shang, Xiangyu Jin and \\ Xiaorui Wang \\ School of Electronics and Information Engineering, Tianjin Polytechnic University, \\ TianJin, 300387, China \\ E-mail: zhengyu@tjpu.edu.cn
}

\begin{abstract}
Amplitude of digital signal attenuates seriously with the increase of probing depth, transmission rate and random noise, because channel impedance parameters of deep-sea abandoned profiler measuring instrument is time-varying in the process of transmission and it will seriously affect the stability of signal transmission in the ASK frequency band transmission. This paper first expounds the method of ASK amplitude demodulation and optimizes the transmission channel model. Secondly, this paper analyzes the variation of XCTD channel impedance parameters in the range of $2000 \mathrm{~m}$ detection. And then, the method focuses on the effects of random noise, XCTD channel time-varying parameters and the transmission frequency on demodulation signal. It comes to conclusion that noise is the main factor affecting the stability of ASK transmission. Meanwhile, this paper analyzes in detail the size of error rate of demodulated signal in great depth measurement. In the process of ASK band transmission in the detection range of $2000 \mathrm{~m}$, the accuracy of signal transmission can be improved to some extent by selecting the band pass filter rightly. In the kind of transmission condition, the noise magnitude less than $-50 \mathrm{~dB}$ is most suitable for ASK band transmission mode.
\end{abstract}

Keywords: Expendable Profile Measuring Instrument; Time-Varying Channel; Amplitude Modulation; Error Rate; Transmission Frequency; Probing Depth.

\section{Introduction}

The Expendable Conductivity Temperature Depth (XCTD) profiler measurement equipment is one of the most important instruments for deep-sea exploration[1-2] and it consists of a launcher, an underwater probe, a transmission channel and a signal receiver. After the probe launched into seawater by the launcher, it measures the marine environment parameters and transmits the data to signal receiver to be processed through a 2000-meter transmission line. 
The signal can't be transmitted stably in the channel because of the influence of the time-variability of XCTD channel impedance parameters and the sea noise [3], so it is very important to improve the stability of the signal in the variable-parameter channel [4]. The baseband transmission has long been unable to meet the demand of deep-sea measurement even though ZhiCheng Jia [5] used baseband transmission based on Manchester encoding. As the main communication technology, the application of ASK modulation in the transmission system of underwater data has been reported [6]. However, with the increase of the probing depth, the transmission frequency and the random noise of the channel, the error rate of the received signal is greatly improved[7]. However, nobody has made deep analysis of the problem. This paper first establishes the model of the transmission channel, then selects the key measuring point to analyze the influence of channel impedance parameters, transmission frequency and random noise on ASK demodulation. At last, as shown in Fig(1), the size and the reason of the error rate are analyzed to find out the suitable conditions, which is suitable for the XCTD channel.

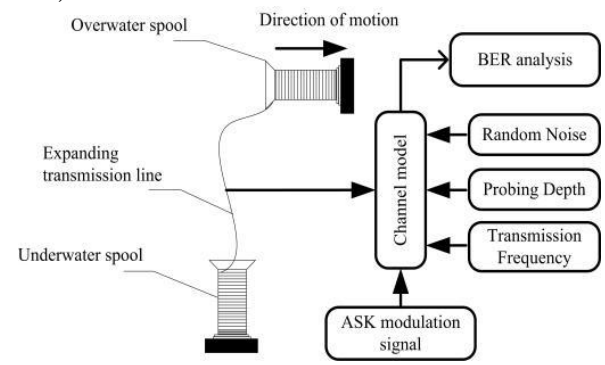

Fig. 1 The XCTD channel characteristics and the influence on ASK modulation transmission

\section{The Establishment of Channel Transmission Model}

According to the variation rule of each parameter in the pay-off process, the previous circuit model is optimized as shown in Fig (2). The XCTD channel consists of underwater spool, expanded transmission line and overwater spool, so the channel circuit model established is made up of three sub modules. L1, $\mathrm{R} 1$ and $\mathrm{C} 1$ are the circuit parameters of the underwater spool, and L2, R2 and $\mathrm{C} 2$ are those of the overwater spool. R3 and R4 are the resistance of the released parallel lines and C3 is the distributed capacitance of it. The value of loading terminal resistance R0 is $1 \mathrm{M} \Omega$. In this paper, the impact of the channel on ASK modulation is analyzed based on the established channel model. 


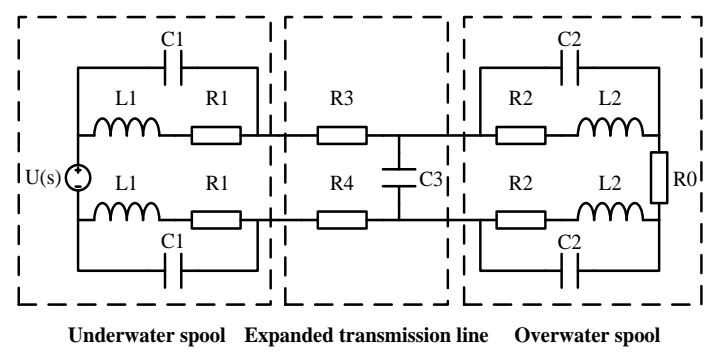

Fig. 2 Simplified channel circuit model

We take advantage of the mesh current method to calculate the channel model in the s-domain. Assuming that the mesh current of the underwater coil and the expanded transmission line is $I_{1}$ and the mesh current of the overwater coil is $I_{2}$, the calculation equation can be obtained as shown in formula(1).

$$
\left\{\begin{array}{l}
U(S)=\left(2 X_{1}+2 R\right) I_{1}+\frac{1}{S C_{3}}\left(I_{1}-I_{2}\right) \\
\left(2 X_{2}+R_{0}\right) I_{2}+\frac{1}{S C_{3}}\left(I_{2}-I_{1}\right)=0 \\
U_{O}(S)=I_{2} R_{0}
\end{array}\right.
$$

$X_{1}$ and $X_{2}$ are the complex impedances which are produced by the single enameled wire winding underwater coil and overwater coil. The expression is separately shown in formula (2) and (3).

$$
\begin{gathered}
X_{1}=\frac{L_{1} S+R_{1}}{L_{1} C_{1} S^{2}+R_{1} C_{1} S+1} \\
X_{2}=\frac{L_{2} S+R_{2}}{L_{2} C_{2} S^{2}+R_{2} C_{2} S+1}
\end{gathered}
$$

So the final transfer function can be solved as:

$$
H(S)=\frac{R_{0}}{b_{3} S^{3}+b_{2} S^{2}+b_{1} S^{1}+b_{0}}
$$

In the formula (4): 


$$
\left\{\begin{array}{l}
b_{3}=4 L_{1} L_{2} C_{3} \\
b_{2}=2 C_{3}\left[L_{1}\left(R_{0}+2 R_{2}\right)+L_{2}\left(2 R_{1}+2 R\right)\right] \\
b_{1}=2 C_{3}\left[\left(R_{1}+R\right)\left(R_{0}+2 R_{2}\right)\right]+2\left(L_{1}+L_{2}\right) \\
b_{0}=R_{0}+2 R_{1}+2 R_{2}+2 R
\end{array}\right.
$$

\section{The Analysis of the Influence of Channel on ASK Transmission}

From the above analysis we can know that probing depth, signal transmission frequency and the level of random noise are the major problems that affect the accurate transmission of XCTD channel signal. The lowest transmission frequency of the signal is $300 \mathrm{~Hz}$. Therefore, we analyze the pay-off process of $2000 \mathrm{~m}$ with the frequency of $300 \mathrm{~Hz}, 600 \mathrm{~Hz}, 800 \mathrm{~Hz}$ and $1200 \mathrm{~Hz}$. Because the key problem of the effect of random noise and burst noise on ASK modulation output is the amplitude, the paper analyzes the effect of different level of noise on ASK modulate. With the level of the random noise increasing, the carrier carries less and less effective signal. So we mainly choose the level of noise from $-80 \mathrm{~dB}$ to $10 \mathrm{~dB}$.

\section{The Influence of the Factors on ASK Frequency Band Transmission}

According to the feature point we selected, we analyze the error rate of ASK demodulation in different transmission conditions, which is shown in Fig (3).
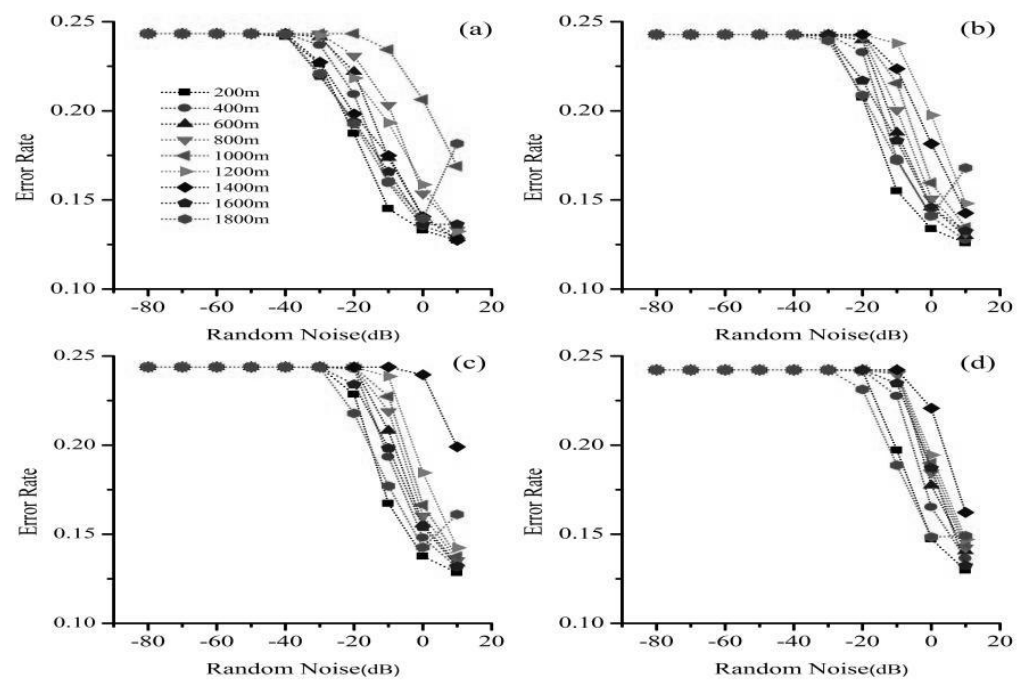
Fig. 3 The effects of probing depth, transmission frequency and noise on ASK frequency band transmission(a) the transmission frequency is $300 \mathrm{~Hz}$;(b) the transmission frequency is $600 \mathrm{~Hz}$; (c) the transmission frequency is $800 \mathrm{~Hz}$; (d) the transmission frequency is $1200 \mathrm{~Hz}$

It is concluded that the change of the noise level is the main factor affecting the accuracy of ASK band transmission through the analysis of Fig (3). When the transmission frequency increases from $300 \mathrm{~Hz}$ to $1200 \mathrm{~Hz}$, the error rate is relatively stable and reaches the maximum value within the scope of the small noise level. The maximum fluctuating range of error rate is $0.6 \%$ with the increase of the transmission frequency. The error rate increases with the increase of transmission frequency within the scope of the large noise level. The noise level that keeps error rate relatively stable increases from $-50 \mathrm{~dB}$ to $-30 \mathrm{~dB}$ and the depth that keeps error rate increasing to the maximum with the increase of probing depth increases from $1000 \mathrm{~m}$ to $1400 \mathrm{~m}$.

\subsection{The ASK frequency band transmission}

The bandpass filter selected has great significance on the accuracy of ASK band frequency transmission. The curve diagram of error rate when the transmission frequency is $300 \mathrm{~Hz}$ and $1200 \mathrm{~Hz}$ with selecting the bandpass filter rightly is shown in Fig(4).

Through the analysis of the experimental data, it is concluded that in the $2000 \mathrm{~m}$ transmission process, the error rate increase with the increase of the noise level. In addition, the maximum value is 0.240 . At the same time, the accuracy of ASK frequency band transmission is the highest.
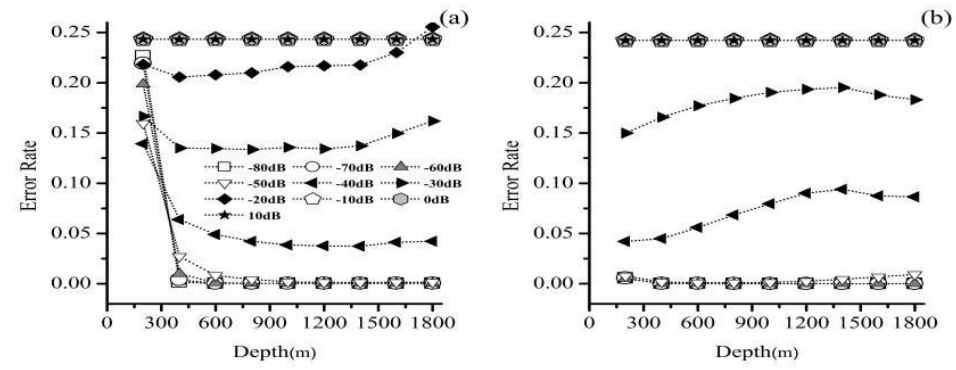

Fig. 4 The curve diagram of the error rate(a) the transmission frequency is $300 \mathrm{~Hz}$;(b) the transmission frequency is $1200 \mathrm{~Hz}$

\section{Conclusion}

As a typical expendable conductivity temperature depth profile measurement equipment, XCTD has great significance on the ocean surveying. The conclusion that random noise, transmission frequency and the changes of XCTD 
time-varying channel parameters all result in signal amplitude attenuation is obtained through the analysis of the reasons of signal amplitude attenuation during the whole process of pay-off. When the noise level is small, The accuracy of ASK frequency band transmission is the highest in this transmission condition. the conclusions can make us better understand the influence of the XCTD channel on signal transmission performance and improve the accuracy of measurement data and stability of signal transmission has important significance.

\section{Acknowledgments}

This research was supported by the Research Program of Application Foundation and Advanced Technology of Tianjin (No.14JCYBJC16300). The National Undergraduate Training Programs for Innovation and Entrepreneurship of China(No.201510058035).

\section{References}

1. Uehara, K., S. Kizu, K. Hanawa, et.al. 2008: Estimation of heat and freshwater transports in the North Pacific using high-resolution expendable bathythermograph data.Geophys.Res.,113,C02014, doi:10.1029.

2. Shoichi Kizua, Hiroji Onishib, Toshio Sugaa, et.al. 2008:Evaluation of the fall rates of the present and developmental XCTDs. Deep-Sea Research,55,571 586,doi:10.1016/j.dsr.2007.12.011.

3. LIU H S, HU Y, YIN Y X, et.al. 2013 :Shallow Water Body Data Processing Based on the Seismic Oceanography. J. Ocean Univ.China,12,319-326,doi: 10.1007/s11802-013-2100-5.

4. Zheng Y, Fu X H, Zhao X, et.al. 2014: The Method of Improving the Phase Stability of Time-Varying Channels of Vessel-Mounted XCTD Profilers .Ocean Technol,33, 3(in Chinese), doi: 10.3969/j.issn.10032029.2006.01.024.

5. Jia Z C, Yu X S, Ni J J, et.al. 2010: Design and Realization of Data Transmission Circuit for XCTD Profiler.Ocean Technol.,29,1 (in Chinese), doi: 10.3969/j.issn.1003-2029.2010.02.001.

6. Zhou Q R, Liu H Y, Ma J, et.al. 2014:A smooth evolution scheme to remodulated WDM-PON based on POLSK/ASK orthogonal modulation. MICROW.OPT.TECHN.LET.,56,2598-2601,doi:10.1002/mop.28651 
7. Zheng Y, Zhao X, Li J, et.al. 2014: Analysis of time-varying channel effects on transmission performance of deep-sea abandoned measuring instrument. Acta Phys. Sin.,63 ,40507 (in Chinese), doi: 10.7498/aps.63.040507.

8. Zheng Y, LiH Z, Liang J, LiuN,2013: Modeling and calculation of distributed capacitance between the transmission lines of underwater XCTD profiler. Marine Science Bulletin, 32, 332-337. 